\title{
Representaciones sobre la adolescencia en Golfito: Sexualidad y reproducción según el sistema público de salud costarricense
}

Recibido: 5 de febrero 2020

Revisado: 17 de abril 2020

Aprobado: 11 de marzo 2021

Mauricio López Ruiz

Costarricense. Doctor en sociología por la Universidad de Costa Rica (Costa Rica). Labora en el Instituto de Investigaciones Sociales de la Universidad de Costa Rica en temas relacionados con la sociología de la salud en materia de desarrollo de políticas y derechos sociales. Correo electrónico: mauricio.lopez@ucr.ac.cr

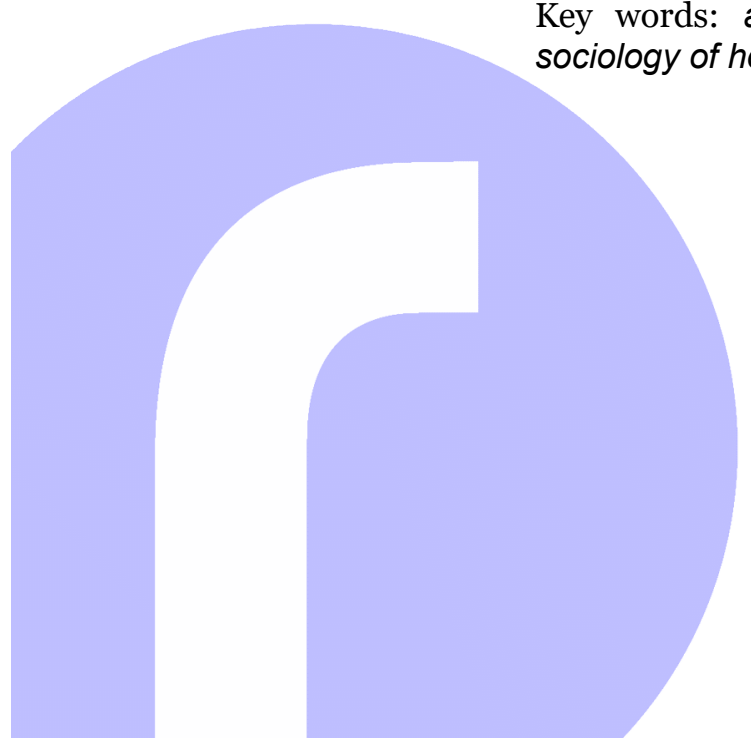

https://revistas.uned.ac.cr/index.php/rupturas cc) (i) (2)
Resumen: En este artículo, se analiza cómo la salud sexual y reproductiva ha sido problematizada en el sistema público de salud costarricense, en el escenario de implementación de la Iniciativa Mesoamérica en la zona de Golfito. En las siguientes páginas, se desarrolla la tesis de que la persona adolescente es juzgada desde la patología y la anormalidad, resaltando fallos personales (afectivos o identitarios) o fallos en la socialización (reproducción de hábitos aprendidos de familiares y grupos de pares). Como consecuencia, el reconocimiento de derechos parte de una concepción negativa sobre la adolescencia y la salud. Este estudio es de corte cualitativo, y fue fundamentado tanto por medio de análisis documentales, como de entrevistas y grupos focalizados realizados a personal en salud pública.

Palabras clave: adolescencia; salud sexual; derechos en salud; sociología de la salud

Representation on Adolescence in Golfito: Sexuality and Reproduction According to Costa Rica's Public Healthcare System

Abstract: This article analyses how sexual and reproductive health has been constructed as a policy problem in Costa Rica's Public Healthcare System, in the context of implementation of the Mesoamerica Initiative in Golfito region. It will be argued that adolescents are seen from the pathology and anormality focusing on personal fails (their affectivity and identity) as well as socialization fails (relative to family and peer groups). As a result, their rights recognition is based on a negative stand. The study is qualitative -document analysis, as well as interviews and focus groups conducted to public healthcare officials.

Key words: adolescence; sexual and reproductive health; health rights; sociology of health 
1. Los derechos sociales, según T.H, Marshall (1992), constituyen una especie de garantías organizativas e institucionales diseñadas para corregir injusticias y desigualdades que atentan contra los chances individuales de disfrutar de una vida segura y digna. Sobre el desarrollo de derechos en salud para población adolescente, véase López-Ruiz (mimeo) y Rodríguez (2014).

2. Se le entiende como la capacidad individual de las personas para alcanzar un buen estado físico, emocional y mental ligado a la vida sexual, así como la capacidad colectiva de alcanzar formas de organización social que le permitan a la gente establecer prácticas y relaciones sexuales placenteras y seguras, libres de coerción, discriminación y violencia (OMS, 2006: 5). Véase Coleman (2011), Correa, Petchesky y Parker (2008), Lottes (2013) y Mitchell, Wellings, y Zuurmond (2012: 74).

\section{Introducción}

La adolescencia es un momento clave, determinante en mucho de lo que nos depararán los años que nos resten por vivir. Ciertamente, se encuentra en juego no solo en términos del nuestro desarrollo y bienestar físico, sino, además, nuestro crecimiento personal y emocional. Por ello, no deja de sorprender la escaza atención especializada que ofrecen a este curso de vida sistemas públicos de salud como el costarricense. En contraste con la atención integral dada el tema de la niñez (en la cual se despliega un enorme esfuerzo institucional en cuanto a prestación de bienes y servicios), el de adolescencia suele ocupar un lugar secundario en las agendas de política en salud, dando lugar a acciones más bien cortoplacistas o a estrategias dirigidas a la atención de una determinada problemática.

Uno de los pocos temas que ha sido trabajado de manera continua durante las últimas tres décadas, a propósito de la adolescencia, es el de salud sexual y reproductiva. A pesar de los altibajos presentes en la formulación y puesta en práctica de acciones políticas, ha logrado incluso obtener el estatus formal de un derecho social. ${ }^{1}$ Con ello, se ha hecho eco de políticas regionales promovidas por la Organización Panamericana de la Salud (OPS) o la Organización Mundial de la Salud (OMS), en que el derecho a la salud sexual y reproductiva aparece reconocido, lo cual implica, entre otras cosas, el darle forma a una cultura cívica que fomente su discusión en el marco de parámetros de mutuo respeto. ${ }^{2}$

En el sistema público de salud costarricense, el desarrollo actual de dicho tipo de políticas ha recibido un significativo impulso con la puesta en marcha, en el 2014, del Proyecto Mesoamérica 2015, que luego se denominará Iniciativa Mesoamérica (BID 2013), y de dos instrumentos legales y de planificación establecidos en el 2015, a saber, la Norma Nacional para la Atención Integral de la Salud de las Personas Adolescentes: Componente de Salud Sexual y Salud Reproductiva (Ministerio de Salud 2015a) y la Normativa Interinstitucional e Intersectorial para la Prevención y Atención del Embarazo en la Adolescencia (Ministerio de Salud 2015b). Luego de concluir su segunda fase de implementación en el 2018 (desarrollada en once cantones), las acciones se estarían generalizando al resto del país, en tanto deja de enfocarse únicamente en el tema de embarazo adolescente para abarcar de manera general el de la salud sexual de la población adolescente (Ministerio de Salud 2014a: 25-28; Ávalos 2015).

Allende la importancia de lo escrito en esos instrumentos normativos y de gestión, el reconocimiento de facto de este derecho dependerá en buena medida de las concepciones o representaciones de los profesionales en salud acerca de la persona adolescente. Esta será la base del trato médico y humano que a futuro recibirán sus jóvenes pacientes. Justamente, el presente artículo versa sobre dichas concepciones y representaciones, de la manera 
en que ellas son reproducidas por parte de personal de la Caja Costarricense de Seguro Social (CCSS) y el Ministerio de Salud (MINSA) en el cantón de Golfito, tanto en su manera de problematizar el tema de salud sexual y reproductiva, como en las acciones de tipo informativo que nutren sus estrategias preventivas.

El argumento central de esta investigación señala que, a pesar de los avances formales registrados en materia de reconocimiento de derechos, la persona adolescente sigue valorándose en términos negativos, tanto en su actuar como en su moralidad. La pista inicial que se obtuvo surgió en medio de una de las sesiones de grupo focal efectuada en Golfito, con representantes del sistema de salud, cuyo objeto era charlar sobre los avances de la Iniciativa Mesoamérica. Ahí se afirmó lo siguiente:

Hemos visto que el inicio de la sexualidad adolescente es problemático, quizá más de lo que debería ser. Entonces, lo que nos toca es enseñarles a estos chicos es que tener sexo seguro, o no tenerlo del todo, es la única opción responsable... Una vez entregado el mensaje, que tomen sus decisiones y asuman sus riesgos (Grupo focal, representantes sistema de salud).

En este texto se encuentran presentes dos claves interpretativas de gran importancia. La primera, sería la categorización negativa que se hace de la persona adolescente. La segunda, tiene que ver con la labor educativa asumida por quien presta servicios en virtud de estas categorizaciones, que permite valorar la manera en que se reconoce su derecho a la salud desde el tema de riesgo. Tal como se explicará más adelante, el encuadre negativo con el cual se piensa a la adolescencia facilita el asumir que sus estados de salud son el producto de un fallo personal (digamos, una carencia afectiva) o un atributo individual de una socialización defectuosa (la reproducción de un mal hábito aprendido en casa).

Tales valoraciones en negativo de los adolescentes, y por extensión de la adolescencia en tanto ciclo de vida, permea de manera inevitable la manera en que al final de cuentas se conciben las intervenciones en salud pública. El caso del desarrollo de la Iniciativa Mesoamérica en Golfito permite explorar con más detalle lo anterior.

\section{Aspectos conceptuales y metodológicos}

Como fue mencionado anteriormente, este artículo describe y analiza ideas acerca de la persona adolescente, reproducidas por funcionarios (hombres y mujeres) clave en el desarrollo de la Iniciativa Mesoamérica en la región $\mathrm{Pa}$ - 
cífico Sur. Dichas ideas han sido conceptualizadas en términos de concepciones o representaciones, es decir:

Construcciones simbólicas individuales o colectivas a las que los sujetos apelan o las que crean para interpretar el mundo, para reflexionar sobre su propia situación y la de los demás y para determinar el alance y la posibilidad de su acción histórica (Vasilachis 2014: 265).

Las preguntas que orientaron la investigación giraron en torno a las temáticas adolescencia, salud y sexualidades. Estas temáticas se desarrollaron en función de tres cuestiones centrales en el diseño de toda política pública: a) aclarar en qué consiste el problema a tratar; b) cómo se explican sus causas; y c) qué soluciones deberían implementarse. Dichas cuestiones, como han sugerido diversos autores (Béland y Cox 2011; Fischer y Gottweis 2011), constituyen una primera base de sentido para las labores de implementación y evaluación que tienen lugar en torno a las políticas.

En la recolección de la información, se contó con la participación de once funcionarias y funcionarios de salud, cuya participación ha sido vital en el desarrollo de estas acciones de política para el caso del cantón de Golfito. Entre ellos se cuentan: un médico, Área Rectora de Salud del Ministerio de Salud en Golfito; dos médicos, representantes de la Dirección Regional de Rectoría de la Salud Brunca, Ministerio de Salud; un trabajador social CCSS Golfito; así como seis representantes de los Equipos Básicos de Atención Integral en Salud (EBAIS) de este cantón (de los cuales se hablará en líneas siguientes). A efectos de respetar el anonimato de las personas participantes en este estudio, no se proporcionan sus nombres, y, además, se ha optado por utilizar el género masculino para su identificación.

Se llevaron a cabo dos series de entrevistas semi-estructuradas entre el 2017 y el 2018. Como complemento de lo anterior, también se efectuaron dos grupos focales, con participaciones de funcionarias y funcionarios de la CCCSS y del MINSA en el 2017, provenientes de la región Brunca, de la cual forma parte Golfito, además de otras poblaciones relevantes como Coto Brus, Puerto Jiménez, y Pérez Zeledón, donde también fue implementada la Iniciativa Mesoamérica. Las conversaciones con estas personas permitieron obtener una perspectiva más general del trabajo en equipo que se ha estado llevando a cabo por parte del sistema de salud en torno a la atención de la salud sexual y reproductiva de la persona adolescente.

Los contenidos de las entrevistas y de los grupos focales se trabajaron utilizando como orientación metodológica la teoría fundamentada (Birks y Mills, 2011). Con ayuda del programa Nvivo 10 (que facilitó el ordenamiento lexicográfico y su comparación intertextual), las entrevistas fueron segmentadas en dos grandes familias de códigos. Al cruzarse, permitieron 
configurar una matriz que facilitó el ordenamiento y estudio de la información de los textos y transcripciones, tal y como se muestra en la tabla 1:

Tabla 1. Matriz de representaciones presentes en entrevistas y diseño de políticas

\begin{tabular}{|c|c|c|c|}
\hline \multirow[b]{2}{*}{$\begin{array}{l}\text { Objeto de la } \\
\text { política }\end{array}$} & \multicolumn{3}{|c|}{ Cuestiones a resolver en el diseño de políticas } \\
\hline & $\begin{array}{l}\text { Problema } \\
\text { relacionado } \\
\text { con: }\end{array}$ & Causado por: & $\begin{array}{l}\text { Cuya solución } \\
\text { depende de: }\end{array}$ \\
\hline Adolescentes & $\begin{array}{l}\text { Riesgo de } \\
\text { embarazo, y } \\
\text { de contraer } \\
\text { ITS } \\
\text { Riesgos }\end{array}$ & $\begin{array}{l}\text {-Inmadurez e } \\
\text { irresponsabilidad } \\
\text {-Consumo de drogas y } \\
\text { violencia }\end{array}$ & $\begin{array}{l}\text { Educación: cambio } \\
\text { de conductas }\end{array}$ \\
\hline Salud & $\begin{array}{l}\text { Riesgos } \\
\text { asociados a la } \\
\text { salud materna } \\
\text { y transmisión } \\
\text { de ITS }\end{array}$ & $\begin{array}{l}\text {-No utilización (o mala } \\
\text { utilización) de métodos } \\
\text { anticonceptivos }\end{array}$ & $\begin{array}{l}\text { Educación: uso } \\
\text { correcto de métodos } \\
\text { anticonceptivos }\end{array}$ \\
\hline Sexualidades & $\begin{array}{l}\text { Riesgos } \\
\text { asociados a } \\
\text { relaciones } \\
\text { coitales } \\
\text { (heterosexual } \\
\text { es) }\end{array}$ & $\begin{array}{l}\text {-Inicio temprano de } \\
\text { relaciones coitales sin } \\
\text { supervisión familiar } \\
\text {-Tener múltiples parejas } \\
\text { sexuales } \\
\text {-No tener relaciones } \\
\text { afectivas de largo plazo } \\
\text {-Cambio en valores } \\
\text { cristianos relativos al } \\
\text { noviazgo, la familia } \\
\text { nuclear, la maternidad y la } \\
\text { paternidad }\end{array}$ & $\begin{array}{l}\text { Educación: } \\
\text { abstinencia, valores } \\
\text { familiares cristianos, } \\
\text { y protección } \\
\text { mediante métodos } \\
\text { anticonceptivos }\end{array}$ \\
\hline
\end{tabular}

Fuente: elaboración propia (2020).

Como puede apreciarse, los ejes de la tabla obedecen a las familias de códigos. En su interior, lo que se observa son las representaciones (códigos, desde el punto de vista de la teoría fundamentada), que en su conjunto dieron forma al discurso sobre salud sexual y reproductiva de la persona adolescente construido. En el cuarto y quinto apartado del presente artículo, se han seleccionado citas que ejemplifican este discurso.

\section{Antecedentes}

Golfito es un cantón de la provincia de Puntarenas, de enorme diversidad biológica y un gran potencial turístico aún por desarrollar, dada la existencia de un bosque tropical húmedo. Se ubica al sur del país, en una zona que exhibe distintos escenarios geográficos, contando zonas montañosas y por- 
tuarias, parques nacionales y reservas naturales, así como territorios indígenas en la zona de Comte Burica y Osa, hogar de la población ngäbe. Limita con importantes comunidades del llamado Sur-Sur, como Buenos Aires o Coto Brus, al igual que Panamá; es, de esta manera, una zona fronteriza que reporta importantes flujos migratorios. Asimismo, su posición geográfica estratégica se debe no solamente a la existencia de un aeropuerto local, sino también al Golfo Dulce, que le brinda a su población una salida al mar, y un característico clima caluroso y lluvioso que se hace sentir a lo largo de gran parte del año.

Con una población que ronda los 40 mil habitantes, según datos obtenidos del último Censo Nacional de Población, Golfito constituye una las poblaciones más importantes de esta zona (PEN-INEC 2013: 164). Se encuentra dividido en cuatro distritos, a saber: Golfito Centro, Puerto Jiménez, Pavón y Guaycará (conocida comúnmente como Río Claro). Históricamente, el desarrollo económico y social de esta zona dependió de la actividad bananera. Luego de que las compañías bananeras abandonaran la región en la década de los años ochenta del siglo pasado, la economía del lugar ha dependido de actividades tradicionales tales como la explotación de maderas, la agricultura, la producción pecuaria, o la actividad pesquera, lo mismo que de la incursión en el desarrollo de un mercado de servicios, en virtud del establecimiento de una zona de libre comercio en 1986; y, más recientemente, la fallida creación de una marina (Marín, Bartels y Abarca 2011).

En cuanto a su desarrollo social, dicha zona constituye la más pobre y desigual de Costa Rica. Si bien, por una parte, la población en general presenta buenos niveles de alfabetización, a un nivel elemental, únicamente $27 \%$ había logrado, para el 2011, la educación secundaria completa (comparado con $39 \%$ que reportaba el promedio nacional). Por otra parte, $20 \%$ de hogares afirmaba tener recursos insuficientes para vivir, lo cual duplica los niveles que se reportan (en promedio) a escala nacional. Ligado a lo anterior, más de la mitad de sus hogares reportaron necesidades básicas insatisfechas, al tiempo que $23,7 \%$ no estaba cubierta por el seguro social, situacion que complica su integración al sistema público de salud (PEN-INEC 2013: 164).

Con respecto a dicho sistema, cabe indicar que la independencia administrativa y gerencial del Área de Salud de Golfito sucedió recientemente, en el 2000. En ese momento, la prestación de servicios (en atención primaria) se apoyó en tres EBAIS: Puerto Jiménez, Río Claro y Conte (que se sumaron a la prestación de servicios brindada a nivel secundario por parte del Hospital de Golfito). Hoy, también cuentan con EBAIS las comunidades de La Palma, Alamedas, San Martín, San Andrés, y Villa Briceño (entrevistas, representantes Dirección Regional de Rectoría de la Salud Brunca, Ministerio de Salud). En materia de atención secundaria, la región cuenta con el Hospital de Golfito, el cual presta servicios en ginecología, obstetricia, pediatría y medicina general; tiene en su haber también herramientas mínimas para la realización de diagnósticos, tales como rayos $\mathrm{X}$ y laboratorio clínico (Ministerio de Salud, 
2013: 68). Por ello, la atención médica a la cual podría acceder la población de Golfito es básica, tanto en el primero como segundo nivel de atención.

Ahora bien, en cuanto al perfil epidemiológico general de la población, según la última sistematización de datos que se encuentra disponible en el documento Análisis de Situación de la Producción Social de la Salud (conocido como ASIS) del año 2012 (Ministerio de Salud 2012), se tendría entre las principales causas de consulta aquellas relacionadas con condiciones de vida inadecuadas para el bienestar humano, como, por ejemplo, infecciones de las vías respiratorias superiores, asmas, parasitosis intestinales, patologías gastrointestinales, patologías dermatológicas, o trastornos del sistema urinario, al igual que casos de urgencia en materia de odontología general. En este sentido, la información recolectada no distinguía para estos casos la situación de los grupos etarios que componen a dicha población (Ministerio de Salud, 2013: 72-73; entrevista: trabajador social CCSS Golfito).

Con respecto al tema de salud sexual y reproductiva en población adolescente, los únicos datos consignados se relacionaron con la cantidad de nacimientos dados en la región, con altas tasas de natalidad (21,7 en Golfito, mientras que en el resto del país se reportaba un promedio de 16,3), así como un número de embarazos entre adolescentes que representaba 24\% del total de casos de gravidez (Ministerio de Salud, 2013: 105; 41). Asimismo, solo se hizo una breve referencia a la aparición de dos casos de Virus de la Inmunodeficiencia humana (VIH) en ese tiempo, así como la necesidad de contar con más datos sobre este tema (Ministerio de Salud, 2013: 150). Según las entrevistas realizadas para el presente trabajo, esta falta de información resulta preocupante en la región; y constituye una muestra del abandono al cual por muchos años había venido siendo objeto la población adolescente (entrevistas: representantes de la Dirección Regional de Rectoría de la Salud Brunca, Ministerio de Salud; trabajador social CCSS Golfito).

Ante un escenario tal, el desarrollo de acciones como la Iniciativa Mesoamérica ha resultado de gran relevancia. Su puesta en marcha en el 2014 «no pudo ser más que bienvenida» en momento en los cuales únicamente era posible «especular e ir desarrollando acciones un tanto improvisadas», a efectos de atender las necesidades de esta población (Grupo focal: representantes del sistema de salud). Como su nombre lo sugiere, dicha acción de política inició siendo una iniciativa de alcance regional; se contó en una primera fase con el apoyo financiero de la Fundación Bill \& Melinda Gates, el Instituto Carlos Slim de la Salud, el Gobierno de España y el Banco Interamericano de Desarrollo (BID) (el cual funge como administrador). Inicialmente, su fin era el ayudar a reducir brechas en materia de desigualdad social, en el caso costarricense, tomando como eje central la salud materna (y la meta de bajar las altas tasas de natalidad adolescente en cantones con altos niveles de pobreza) (BID: 2013). 
3. Grosso modo, ambos documentos sintetizan el marco de derechos sobre el cual se entiende la atención de la salud sexual y reproductiva, incluyendo una directriz organizativa en relación con el trabajo de instituciones como el Patronato Nacional de la Infancia (PANI), el Ministerio de Educación Pública (MEP), así como los Centros de

Educación y Nutrición / Centros Infantiles de Nutrición y

Atención Integral (CEN-CINAI).
Antes de su puesta en marcha en Golfito, existía una serie de acciones desarticuladas, muchas veces efectuadas a título personal por algunos funcionarios comprometidos con el bienestar de la persona adolescente (entrevistas: representantes de la Dirección Regional de Rectoría de la Salud Brunca, Ministerio de Salud; trabajador social CCSS Golfito). Había ocasiones en las cuales el personal de salud aprovechaba para realizar talleres sobre educación sexual en centros públicos de educación secundaria [incluyendo temas como métodos anticonceptivos y profilácticos 0 transmisión de infecciones de transmisión sexual (ITS)], y hablar sobre alguna otra problemática de salud prioritaria para la zona. En esos momentos, se aprovechaba también para aplicar herramientas de detección de riesgos, que pudieran remitirse al personal de psicología o trabajo social de la zona. (entrevista: trabajador social CCSS Golfito).

A partir del desarrollo de la Iniciativa Mesoamérica, se ha buscado institucionalizar este tipo de prácticas, dándoles un marco de sentido fundado en el tema de derechos humanos. Entre ellos, se hallan los siguientes: a) realizar análisis de la situación de salud de la persona adolescente; b) garantizarle una atención diferenciada dentro de un espacio físico habilitado para tales fines; c) respaldar los servicios con personal calificado en este grupo de edad; d) aplicar instrumentos adecuados de evaluación de riesgos en salud sexual y reproductiva; e) poner a disposición de las personas métodos anticonceptivos y de protección; f) establecer estrategias educativas individuales y grupales; y g) implementar una modalidad de atención diferenciada para adolescentes embarazadas, madres y padres adolescentes (Ministerio de Salud 2015a: 28-29).

La iniciativa se ha venido desarrollando en dos etapas, de 18 meses cada una; se inició en el 2014. En la primera, se llevó a cabo un trabajo de detección de problemas relativos a la cobertura de la población adolescente, la existencia de barreras en cuanto a la prestación de servicios en salud sexual y reproductiva, las bases de datos existentes, al igual que las estrategias de comunicación utilizadas por el personal de salud pública (entrevistas: representantes de la Dirección Regional de Rectoría de la Salud Brunca, Ministerio de Salud). Durante la segunda, empezaron a regir dos instrumentos normativos y de planificación: la Norma Nacional para la Atención Integral de la Salud de las Personas Adolescentes: Componente de Salud Sexual y Salud Reproductiva (Ministerio de Salud 2015a) y la Normativa Interinstitucional e Intersectorial para la Prevención y Atención del Embarazo en la Adolescencia (Ministerio de Salud 2015b). Ambos fueron diseñados para ser aplicados a escala nacional; Golfito fue uno de los primeros lugares en donde empezaron a operar. ${ }^{3}$

Como puede notarse, con estas normativas se amplía de manera significativa el alcance original de la Iniciativa Mesoamérica, en tanto trasciende el tema de embarazo adolescentes para abarcar el de salud sexual y reproductivo de la población adolescente en general. Una vez finalizadas sus dos fases principales, han podido constatarse una serie de tareas pendientes. Partiendo 
del conjunto de las entrevistas realizadas a los representantes del Ministerio de Salud, es posible resumir y destacar las siguientes:

- Mejorar la capacidad que se tiene desde los EBAIS, de captar población adolescente. Estos espacios constituyen los sitios ideales donde podría darse este contacto, mediante lo que se ha llamado la Consulta de Atención Integral Individual (sería deseable realizarla varias veces a lo largo de la adolescencia).

- Mejorar los mecanismos de convocatoria, para ampliar la cantidad de población que se encuentra recibiendo los beneficios de esta iniciativa, así como establecer un ambiente adecuado para promover el trabajo entre grupos de pares. Además, se plantea trabajar con adolescentes excluidos del sistema educativo, complementando así la atención que se brinda a población escolarizada.

- Continuar fortaleciendo los esfuerzos de prestación de bienes, en este caso, métodos contraceptivos y de barrera. En la región de Golfito, y en otras zonas donde la prestación de servicios se da en medio de enormes carencias materiales, resulta necesario conseguir el financiamiento para la provisión de cinco tipos de métodos (condón masculino, pastilla anticonceptiva, dispositivo intrauterino, solución inyectable mensual, y el implante subdérmico).

- Para el caso de las mujeres embarazadas, resulta vital seguir trabajando junto con otras instituciones de gobierno para evitar su deserción escolar. Esto implica no solo otorgar algún tipo de estipendio económico (condicionado por su desenvolvimiento escolar), sino también la puesta en marcha de un seguimiento a largo plazo que minimice el riesgo social en el cual puedan caer estas personas.

- Fortalecer el trabajo interinstitucional, mediante la creación de perfiles de salud relativos a la población adolescente. Ya para finales del 2018, se pudo avanzar, por primera vez, en la construcción de una base de datos con información de población adolescente en situación de riesgo, gracias al trabajo mancomunado de la CCSS, el MINSA, el MEP y el Patronato Nacional de la Infancia (PANI). Con ello, se inauguró un trabajo interdisciplinario del cual se esperan más avances a futuro.

Por último, como fue mencionado en una de las sesiones de grupo focal sostenida con personal del sistema de salud en Golfito, sería necesario tomar en cuenta que la Iniciativa Mesoamérica, con todas las oportunidades que puede ofrecer, también «ha requerido y requerirá de una labor de tropicalización o adaptación a la realidad local de la región sur-sur». En este sentido, su diseño original respondía a una "visión centralista» que no tomaba en cuenta «los diversos problemas en salud que se enfrenta a nivel local» (se ha establecido una serie de intervenciones prioritarias anteriores), así como demandas que implican «un recargo en recursos disponibles, de tiempos y de capacidad de redoblar esfuerzos organizativos, de funcionarios que incluso ponen 
de su bolsillo para echar a andar procesos» (Grupo focal: representantes del sistema de salud).

\section{La problematización de la salud sexual y reproductiva durante la adolescencia}

Una dimensión del desarrollo de las políticas en salud se relaciona con su planificación y desarrollo operativo. Grosso, ella ha sido descrita anteriormente. Sin embargo, otra dimensión indispensable tiene que ver con la manera en que la idea de adolescencia cobra sentido para quienes tienen a su cargo echar a andar estas políticas. Este apartado y el siguiente desarrollan este tema, mediante la reconstrucción de algunas representaciones subyacentes en sus discursos. Para iniciar, se les solicitó a los participantes de la investigación que explicaran cuál definición general de salud sexual y reproductiva han tratado de promocionar por medio de su trabajo profesional. Sin excepción alguna, en sus narraciones se recuperaron enunciados propios de la definición con enfoque de derechos citada en la introducción del presente artículo, tales como «el no estar condenado a tener que vivir con alguna ITS», «la posibilidad de tener una buena condición física y mental », «la libertad de poder elegir con cuál pareja estar, o en qué momento se desea tener un hijo » o bien, como « el chance de alcanzar una vida plena, de disfrutar de tu sexualidad, sin tener que pasar por experiencias traumáticas o violentas » (entrevistas: representantes EBAIS Golfito).

Ahora bien, al solicitarles que desarrollaran una definición de salud acorde con sus experiencias en las comunidades donde han trabajado, sus discursos empezaron a virar a un enfoque de riesgos, basado en visiones de corte biomédico y heterosexual. Desde este punto de vista, la salud sexual y reproductiva se visualizó como un problema relacionado con la reproducción humana o contagio de enfermedades, confinado al ámbito de relaciones sexuales coitales, las cuales, para el caso de los adolescentes (hombres y mujeres), conducen a embarazos a cortas edades (ese sería el problema principal), o bien, a desarrollar alguna ITS (el VIH, se mencionó vagamente). Por ejemplo, se insistió en la «urgente necesidad de fortalecer los servicios médicos en ginecología y obstetricia » que se estaban ofreciendo, y la relevancia que tiene el hacer campañas en salud dirigidas a enseñarles «a los muchachos que se cuiden más mediante el uso adecuado de un condón», "a temerle a las enfermedades de trasmisión sexual» y "hacerles entender a las chicas jóvenes la importancia que tiene el uso de métodos anticonceptivos, para alejar el peligro de un embarazo no deseado » (entrevista: representantes EBAIS Golfito).

Esta definición más acotada de la salud sexual y reproductiva, basada en el tema de riesgos, tiene un origen práctico. Se vincula, directamente, con los instrumentos de monitoreo utilizados regularmente por los prestadores de servicios en salud a fin de brindar consultas en psicología o trabajo social. Ambos instrumentos han venido siendo utilizados desde hacía más de una década. El primero sirve para discriminar si la persona debe ser remitida a 
servicios de salud infantil o a servicios de medicina general. Se le denomina Test de Tanner, y consiste en una escala de medición del crecimiento corporal que les permite a las personas identificar su respectivo desarrollo biológico sexual desde la niñez hasta la adultez, mediante ilustraciones del tamaño de las mamas, los órganos genitales y el vello púbico (CCSS, s.f.; entrevista: trabajador social CCSS Golfito).

El segundo instrumento, es la Prueba de Tamizaje. Constituye el instrumento de monitoreo sobre riesgos más relevante en el marco del desarrollo de este tipo de políticas. En su primera versión, antes de la Iniciativa Mesoamérica, consistía en un breve cuestionario autoadministrado con veinte preguntas cerradas en donde se preguntaba al adolescente sobre comportamientos individuales tales como el consumo de drogas o el involucramiento en acciones violentas. En la parte referida a salud sexual y reproductiva, se indagaba acerca del número de parejas sexuales que habían tenido, si utilizaban condones, si intercambiaron sexo por dinero o droga, o si habían experimentado relaciones sexuales mediadas por violencia (CCSS, s.f.). La información era recolectada en centros médicos o escolares, y solo fue utilizado para elaborar expedientes clínicos, con el fin de darles seguimiento a casos de embarazo adolescente, o de contagio por ITS (entrevista: trabajador social CCSS Golfito). ${ }^{4}$

Parte de los cambios que trajo consigo la Iniciativa Mesoamérica, consistió en modificar esta prueba y sentar las bases para una mejor sistematización de datos (para el desarrollo futuro de investigaciones epidemiológicas). El nuevo cuestionario amplió la batería de preguntas y su respectiva categorización de riesgos, con temáticas tales como: situación en el hogar (estructura familiar a la cual pertenece y si tiene o no hijos); educación y empleo; vida social (si ha sufrido situaciones de maltrato o abuso, y si participa en grupos socioculturales); salud mental (uso de drogas); imagen corporal; salud mental; y más importante para esta investigación, salud sexual y reproductiva. Sobre esta última temática, se indagó sobre el número de parejas que han tenido los adolescentes, el grado de acercamiento físico, la utilización de anticonceptivos, el historial de embarazos y abortos, el contagio de ITS o $\mathrm{VIH}$; y, por último, si las relaciones sexuales habían sido mediadas por situaciones de violencia o abuso (CCSS 2015). Dependiendo de sus respuestas, las personas son clasificadas en tres niveles de riesgo, a partir de las cuales se decidía el tipo de atención que debía brindarse.

A pesar de que la información obtenida a partir de la Prueba de Tamizaje (en sus dos versiones) no ha sido analizada en su conjunto para el caso de Golfito, se les solicitó a los entrevistados que dieran su opinión acerca de los rasgos característicos que protegían o hacían vulnerable a los adolescentes de la zona frente a este tipo de riesgos. Tomadas en su conjunto, sus respuestas dieron lugar a un discurso en el cual las actuales generaciones adolescentes se encontrarían pasando por lo que, en este artículo, vamos a designar en términos de una erosión generalizada de arreglos institucionales. Dichos arreglos comprenden, en primer lugar, prácticas asociadas al cortejo y el
4. La consulta ofrecida a adolescentes en riesgo nunca fue de carácter obligatorio, lo cual se repite en la actualidad. Dada la falta de recursos, el personal de salud no era capaz de movilizarse para efectuar la consulta en los lugares de habitación de esas personas (entrevistas: representantes EBAIS Golfito). 
noviazgo. Para el caso de adolescentes mayores de 12 años, especialmente mujeres, se señaló la carencia de una adecuada guía familiar, entendida esta en términos de valores religiosos tradicionales (de orden cristiano). Desde este punto de vista, el embarazo se percibía como consecuencia de relacionarse con hombres mayores sin una debida supervisión madres y padres. Igualmente, estas relaciones se entendían no como el producto de relaciones de poder propias de una sociedad patriarcal, sino más bien, dentro de una lógica cultural en que la tradición del noviazgo estaba desapareciendo. En su lugar, a lo largo del Pacífico Sur predominaba la aparición de "parejas juntadas " o de "muchachitas con viejos»" que iban "en contra de la moral cristiana» que " en otros tiempos era propia de esas zonas » (entrevistas, representantes CCSS Golfito).

Un segundo tipo de arreglos corresponde a aquellos relativos a la monogamia social. En este caso, fue mencionado de manera reiterada cómo en la región podía notarse entre la juventud un aumento creciente tanto del "sexo casual» como de "la proliferación de múltiples parejas sexuales»" entre jóvenes. En tanto conductas de riesgo, lo anterior tendría como principales efectos colaterales el contagio de ITS, lo mismo que el surgimiento de embarazos no deseados. Ambas prácticas fueron calificadas como «aventuras» que demostraban la «inmadurez» de los chicos, y su incapacidad de compromiso afectivo de largo plazo. El problema con estas aventuras era que una vez iniciadas las relaciones sexuales «podría terminarse ligado de por vida con otra persona, y con hijos de por medio» o que las secuelas de «las enfermedades venéreas podrían acompañarlos para siempre» (entrevistas, representantes EBAIS Golfito).

Por último, nuestros entrevistados hicieron referencia a los riesgos que implica el no crecer en familias tradicionales (las que obedecen al ideal de papá, mamá e hijas o hijos). El que los adolescentes no estuvieran interesados en establecer relaciones de largo plazo con una única persona era reflejo de procesos de socialización fallidos por el cual los «padres no enseñaron a sus hijos a valorar el matrimonio " o las implicaciones que conllevan la maternidad y la paternidad. Lo anterior deja mal preparadas a los adolescentes para «las responsabilidades que vienen con un recién nacido, o la carga que implica para una pareja el tener hijos». Dicha situación fue entendida como un "efecto esperado» de haber nacido y crecido en «hogares en crisis, llenos de conflictos, inestables. " En estas circunstancias, parecería que estas personas se encontraban condenadas a repetir, de manera cíclica, un destino inadecuado, mediado por distintos tipos de carencias afectivas (entrevistas, representantes CCSS Golfito).

Con respecto a la erosión de los arreglos mencionados y sus riesgos asociados, en la sesión de grupo focal también se mencionó que los mismos se enmarcaba en un contexto general, comunitario, que promueve la generación de estilos de vida poco favorables para la población joven. El crecer en Golfito implica: 
No tener a la mano espacios públicos de recreación y sitios de convivencia para jóvenes que no giren en torno al consumo de alcohol, por ejemplo, o lugares que ofrezcan oportunidades o formas sanas de entretenimiento, como la música, al igual que espacios fuera de la casa que le permitan a un adolescente aprender el valor de la educación [...] más allá de algunas canchas de futbol, la vida gira en torno al depósito libro, al paseo marino, la pesca, o el inventarse caminatas o excursiones a la frontera."

(Grupo focal: funcionarios del sistema de salud).

\section{Educando al adolescente}

En suma, al encuadrar la noción de salud sexual y reproductiva desde una perspectiva de riesgo, enfatizando los problemas de embarazo y contagio de ITS, la persona adolescente fue concebida como un individuo que sufre los efectos de una erosión de arreglos institucionales tradicionales, religiosos, concernientes al matrimonio, la fidelidad, o el noviazgo. Por extensión, en sus discursos su exposición a riesgos se debía también a fallos de conducta individual producto de una socialización deficiente (inmadurez, irresponsabilidad, o visión cortoplacista). Lo anterior permite entender el sentido que fue dado a la principal estrategia preventiva desarrollada por los funcionarios de salud, a saber: las charlas y actividades educativas que acompañaron las acciones de control de riesgos.

Hasta el 2014, momento en el cual el MEP inició la implementación de sus guías oficiales de estudios sobre sexualidad y afectividad, las charlas preparadas por el personal de salud constituían las únicas acciones desarrolladas desde el Estado en materia de educación en salud sexual y reproductiva. Ellas han conocido dos variantes, siendo la primera, la que se utilizaba antes de implementarse la Iniciativa Mesoamérica. Como recuerdan algunos de los funcionarios de la CCSS entrevistados, sus labores de promoción consistían en visitar centros educativos con poblaciones que estuvieran cursando el noveno año de estudios (escolares con una edad promedio de 15 años). Se brindaban exposiciones magistrales, cuyo mensaje central era que los adolescentes se habían convertido en personas "sexualmente reproductivas" y que esta circunstancia "les pondría en riesgo si se descuidaban." Luego, se explicaban aspectos biológicos relativos a la pubertad y el sistema reproductivo, enfatizando en lo sencillo que podría ser el resultar embarazada, o bien, resultar contagiado por alguna "enfermedad venérea" (entrevistas: representantes EBAIS Golfito). 
5. Específicamente, se hizo referencia a materiales producidos por el Programa Conjunto Giro 180, tales como el Modelo de Promoción de Estilos de Vida Saludables y Prevención del VIH y SIDA entre Adolescentes (UNFPA, 2011).
Entre los materiales de apoyo que se utilizaban en estas actividades, se tiene el manual Cómo planear la vida (CCSS 2003) producido en el proyecto Capación a personas facilitadoras para la prevención de embarazos adolescentes desarrollado por la ONG Asociación Demográfica Costarricense en el 2003, así como textos hechos por organismos internacionales como el UNFPA. ${ }^{5}$ Más allá de los temas concretos en este tipo de materiales, al momento de montar las charlas se enfatizaban ciertos "contenidos prioritarios, tales como la planificación de la vida durante la adolescencia, los valores familiares, el trabajo de la paternidad y maternidad," al igual que información relevante sobre contracepción y comportamientos de riesgo asociados al contagio de ITS y VIH. Las exposiciones magistrales basadas en esos textos solían ser complementadas con actividades participativas individuales y grupales, sobre todo de discusión y comprensión de las ideas enseñadas en esas sesiones (entrevistas: representantes EBAIS Golfito).

Con la Iniciativa Mesoamérica, se introdujeron nuevos materiales de apoyo para este tipo de campañas de promoción de la salud. En este caso, debe hacerse la salvedad que los mismos estuvieron dirigidos a población adolescente identificada en situación de riesgo. Como principal apoyo, se incluyó un Manual de Consejería en Salud Sexual y Salud Reproductiva para Personas Adolescentes (Ministerio de Salud, 2014a), así como un Manual de Actividades Educativas Grupales con énfasis en Salud Sexual y Salud Reproductiva para Personas Adolescentes (Ministerio de Salud, 2014b). Estos nuevos manuales se utilizaron en espacios de diálogo entre adolescentes y personal de salud, con la intención de crear procesos de "escucha activa" capaces de favorecer "el cambio individual desde adentro." Dicho cambio, se base en tres principios elementales: debía centrarse en las necesidades y requerimientos de los adolescentes; no tenía que dar lugar a prácticas discriminatorias, en tanto el rol de la persona consejera se concebía mediada por valores como el ser "auténtica, respetuosa, honesta" entre otros; y debería abocarse a "orientar y no dar direcciones" en tanto no suponía, en buena teoría, establecer ningún tipo de imposición (Ministerio de Salud 2014a: 9-10).

De estos materiales educativos, interesa destacar lo siguiente. A criterio de los entrevistados, antes de la Iniciativa Mesoamérica, las representaciones hechas acerca de las sexualidades estuvieron relacionadas más con la enfermedad que el bienestar, con experiencias negativas más que positivas, con la dimensión individual-orgánica que la social. Esto habla del marcado énfasis biomédico que las caracterizaba. Dado que la población adolescente siempre era retratada en términos de una condición de minoría de edad, no sólo civil sino también emocional e intelectual, era tarea del personal médico brindar información para calcular daños y costos asociados a las relaciones íntimas. Aquí, el recurso al miedo de la decadencia orgánica que trae consigo una ITS, fue el medio de persuasión preferido para llevar a los adolescentes a tomar conciencia sobre la importancia de "controlar su cuerpo, al igual que sus emociones" (entrevistas: representantes EBAIS Golfito). 
Con la llegada de los materiales de trabajo de la Iniciativa Mesoamérica, se introdujeron algunas variantes. La representación de la adolescencia como un ciclo de vida regido por la incertidumbre fue conservada (la cual una vez llegada a la adultez será resuelta), sin embargo, se cambió la estrategia persuasiva. No se apeló al miedo como factor de transformación de los adolescentes, sino más bien, a una responsabilidad individual que, en la presente investigación, se clasificó en tres vertientes: cuidarse a sí mismo, cuidarse de sí mismo (de incurrir en conductas de riesgo) y cuidar a los demás. Para el sector salud, el llamado hecho a los adolescentes consistía en insistirles que debían llevar a cabo un "proceso de introspección" que debía permitirles tomar decisiones de una manera racional, dada la importancia que esto tendría "no sólo en sus vidas, sino en las vidas de todos sus seres queridos" (entrevistas: trabajador social CCSS Golfito; representantes EBAIS Golfito).

Ahora bien, desde el punto de vista de los funcionarios de salud, el efecto ideal que deberían tener estas campañas se resume en dos mundos deseables. El primero, el mejor, era uno en el que los jóvenes se protegían mediante la postergación del inicio de sus relaciones sexuales (entendidas en términos de penetración coital). Se pensaba sobre todo en el tema de embarazo adolescente, haciendo eco no únicamente de regulaciones tradicionales de tipo cristiano (acerca de la importancia de la virginidad), sino, igualmente, de "la relevancia del matrimonio en la adultez, como el momento adecuado para la vida sexual" (entrevistas: representantes EBAIS Golfito).

Para justificar lo anterior se utilizaron argumentos diferenciados por género. Para las mujeres adolescentes, el inicio de relaciones sexuales tendría un impacto a nivel biológico y emocional. Todo embarazo adolescente se considera un embarazo de alto riesgo, en tanto no sería sino hasta la adultez que el organismo femenino preparado para los cambios asociados al período de gestación. En cuanto al aspecto emocional, las adolescentes se asumen como personas con una pobre capacidad "de vérselas con todas las repercusiones psicológicas" de la maternidad, máxime, teniendo en cuenta que ellas "aún no han formado sus propios hogares, ni tienen un buen apoyo por parte de sus parejas." En el caso de los hombres, las preocupaciones se desplazaron al terreno económico. Para ellos, las complicaciones del embarazo se vinculan con su dependencia económica. A esa edad, "los chicos deberían ser más inteligentes, y buscar terminar sus estudios en secundaria para tener el chance de encontrar un buen trabajo." En ese sentido, tener una niña o un niño en ese momento sería poco "inteligente," dado que no estarían en capaz de proveerles un ambiente económico seguro, creando una presión financiera a sus respectivas familias (entrevista: trabajador social CCSS Golfito).

El segundo de los mundos ideales visualizados por los funcionarios de salud sería uno en el cual los adolescentes, a pesar de haber iniciado relaciones sexuales, utilizarían métodos profilácticos. ${ }^{6}$ En este caso, se hablaba de fomentar "una sexualidad responsable" que les protegiera (a sí mismos y a los demás) frente al contagio de ITS y VIH mediante la utilización de condones 6. Al igual que en las narraciones acerca del tema de riesgo, de nuevo fueron prácticas heterosexuales las que ocuparon la atención e masculinos (en este punto, las menciones al tema de embarazo fueron he- funcionarios de salud. 
chas de manera secundaria). En este escenario, se dijo que "los hombres deberían ser en todo momento conscientes de las protecciones que brindan los condones," y que las mujeres deberían ser capaces de pedir su utilización al momento de tener sexo, sin importar si "el tipo de pareja era considerado o no el hombre más conveniente" para ellas (entrevista: trabajador social CCSS Golfito).

En el caso de estos dos tipos de escenarios ideales reconstruidos a partir de las narraciones de los funcionarios de salud, la principal clave discursiva detectada fue de tipo adulto-céntrico. En esta parte de las entrevistas, se insistía continuamente que la adolescencia era básicamente una etapa de inmadurez, en muchos sentidos inacaba, de transición. El ser adolescente suponía ocupar culturalmente una posición ambigua entre el mundo infantil y el adulto. Los cambios físicos que trae la pubertad, así como el inicio de relaciones sexuales (o prácticas como el besarse o el magreo), marcaban distancia con el supuesto carácter asexuado de la niñez (acercándoles de esta manera a la adultez). Al mismo tiempo, y esto fue enfatizado en las referencias sobre embarazo, la situación de dependencia en relación con sus familias colocaba a la persona adolescente en un estatus de subordinación semejante al de la niñez (entrevistas: representantes EBAIS Golfito).

En suma, estos mundos ideales que en buena teoría deberían resultar del desarrollo de las campañas informativas ofrecidas por el personal de salud, tienen como sustento conceptual ideas relativas a la necesidad de control de los afectos que debería mostrar la persona adolescente con el fin de librarse de los riesgos que acarrea el embarazo adolescente o el contagio de ITS. Por lo visto en esta investigación, este debate ha dejado poco espacio a la discusión de otros aspectos positivos y edificantes que podrían pensarse en relación con las sexualidades humanas durante el curso de vida adolescente.

Desde luego, como se explicó en la sesión de grupo focal sostenida en Golfito, este abordaje debe también ser entendido como parte de un contexto sociocultural en donde hablar de sexualidades es en sí un tabú:

Es casi que inevitable que las preguntas sobre sexualidad se presten al morbo, hablar de esto es complicado, incluso para uno como madre o padre cuando un hijo te lo pide, y más si está la religión de por medio. También es algo complicado para uno como funcionario en salud, más en esta zona, no sólo porque se debe pensar en una estrategia dirigida a los adolescentes, que no se transforme en un interrogatorio clínico, sino porque no todo el personal de salud está listo para tener la apertura de hablar sobre sexualidad. 


\section{Conclusión}

Como fue expuesto en los apartados anteriores, si bien es cierto el reconocimiento formal del enfoque de derechos constituye un eje central en la Iniciativa Mesoamérica, el lograr su reconocimiento de facto continúa siendo un proceso en construcción, al cual se le debe prestar atención. En el caso de Golfito, la manera en la cual se le ha dado sentido requiere someter a un examen crítico formas comunes de entender la adolescencia, en donde los integrantes de este grupo etario son juzgados desde la patología y la anormalidad, resaltando fallos personales (afectivos o identitarios) o fallos en la socialización (reproducción de hábitos aprendido de familiares y grupos de pares). Como consecuencia, la marcha de este tipo de políticas no se ha desligado de concepciones negativas sobre la adolescencia y la salud basadas en el riesgo.

A partir de estos hallazgos de investigación, habría al menos tres cuestionamientos que podrían hacerse, a efectos de ofrecer oportunidades de mejora para políticas como la Iniciativa Mesoamérica. Primero, es necesario reconsiderar las limitaciones conceptuales que tiene para el reconocimiento de derechos el hacer girar la discusión en torno al riesgo, y más concretamente, en función de dos problemáticas, a saber, embarazo y contagio de ITS. Segundo, es importante dejar de entender dichas problemáticas como un producto exclusivo de malas decisiones individuales, en tanto se las encasilla en ámbitos de responsabilidad moral individual, que dejan poco espacio para discutir acerca de sus determinantes sociales. Tercero, es preciso cuestionarse una y otra vez, ¿cómo puede abrirse el debate sobre sexualidades, a nociones menos conservadores y más apoyadas en la diversidad?

El traer a colación lo anterior tiene la finalidad de recordar que el desarrollo de las políticas sociales no es sólo un asunto de recursos financieros, capacidades organizativas ya instaladas, o voluntades políticas (aunque ellas sean decisivas). Para el caso de la persona adolescente, es vital no sólo dejar de reconocerla a partir de sus patologías biológicas o sociales, sino también reconocer que detrás de sus estados de bienestar o enfermedad individual, existen también responsabilidades colectivas. Para alcanzar este entendimiento, el diseño de políticas y las personas que los llevan a la práctica, deben nutrirse de datos acerca de cómo las nuevas generaciones están viviendo y negociando sus sexualidades y los afectos que a ellas se vinculan, y, sobre todo, las preocupaciones que en ellas se van acuñando.

Para finalizar, hay que decir que el compromiso y la dedicación vistos en los funcionarios de salud pública entrevistados en esta investigación constituye un buen espacio para reflexionar acerca de estos retos, al igual que otros más que aparezcan de camino. De hecho, en esas charlas informales que precedieron a las entrevistas y grupos focales, en repetidas ocasiones se comentó acerca de la necesidad que existía de recibir un asesoramiento y acompañamiento continuos por parte de instituciones como la CCSS y el Ministerio de Salud. ¿Podrá la Iniciativa Mesoamérica no sólo alimentar este renovado impulso, sino, igualmente, servir de base para el desarrollo de postu- 
ras críticas y reflexivas acerca de la salud sexual y reproductiva de la persona adolescente? Ya el tiempo nos lo dirá.

\section{Bibliografía}

Ávalos, Andrea. 2015. "EBAIS de Limón y zona sur trabajarán en prevenir embarazo adolescente." En diario La Nación, 3 febrero. Disponible en: https://www.nacion.com/el-pais/salud/ebais-de-limon-y-zona-surtrabajaran-en-prevenir-embarazo-adolescente/ DVBQIXJJ2BCCTA6YQNZ 427XKKQ

Béland, Daniel y Robert Henry Cox. 2011. "Introduction: Ideas and Politics." En Ideas and politics in Social Science Research, editado por Daniel Béland y Robert Henry, 3-22. Cambridge: Oxford University Press.

BID. 2013. Salud Mesoamérica 2015. Manual Operativo del Proyecto. San José: Banco Interamericano de Desarrollo.

Birks, Melanie y Jane Mills. 2011. Grounded Theory. A Practical Guide. Londres: Sage.

CCSS (s.f.). Test de Tanner e Instrumento de Tamizaje para Adolescentes. San José: Programa de Atención Integral a la Adolescencia.

CCSS. 2003. Cómo planear mi vida. Proyecto capacitación a personas facilitadoras para la prevención de embarazos en adolescentes. San José: Caja Costarricense de Seguridad Social.

CCSS. 2015. Formulario de Evaluación de riesgo. San José: Caja Costarricense de Seguridad Social-Salud Mesoamérica 2015.

Coleman, Eli. 2011. "What is Sexual Health?. Articulating a Sexual Health Approach to HIV Prevention." AIDS and Behaviour, 15 (S1): 18-24.

Correa, Sonia, Rosalind Petchesky y Richard Parker. 2008. Sexuality, Health and Human Rights. Londres: Routledge.

Fischer, Frank y Herbert Gottweis. 2011. The Argumentative Turn Revisited. Public Policy as Communicative Practice. Durham: Duke University Press.

López-Ruiz, Mauricio. Mimeo. En retrospectiva: estableciendo el tratamiento a la salud sexual y reproductiva durante el ciclo de vida adolescente en el marco del PAIA durante los años noventa.

Lottes, Ilsa. 2013. "Sexual Rights; Meanings, Controversies, and Sexual Health Promotion.” Journal of Sex Research 50(3-4): 367-391.

Marín, Bartels y Abarca. 2011. "Introducción." En El Sur-Sur. Trayectorias y perspectivas de una región en proceso de formación 1821-2010, editado por Juan José Marín, Jorge Bartels y Oriester Abarca, 9-22. San José: Sociedad Alquimia 2000. 
Marshall, Thomas Humprey. 1992. Citizenship and Social Class. London: Pluto Press.

Ministerio de Salud. 2012. Análisis de Situación de la Producción Social de la Salud. Golfito: Ministerio de Salud de Costa Rica - Área Rectora Salud Golfito - Dirección de Vigilancia de la Salud.

Ministerio de Salud. 2014a. Manual de Consejería en Salud Sexual y Salud Reproductiva para Personas Adolescentes. San José: Ministerio de Salud.

Ministerio de Salud. 2014b. Manual de Actividades Educativas Grupales con énfasis en Salud Sexual y Salud Reproductiva para Personas Adolescentes. San José: Ministerio de Salud.

Ministerio de Salud. 2015a. Norma Nacional para la Atención Integral de la Salud de las Personas Adolescentes: Componente de Salud Sexual y Salud Reproductiva. San José: Ministerio de Salud.

Ministerio de Salud. 2015b. Normativa Interinstitucional e Intersectorial para la Prevención y Atención del Embarazo en la Adolescencia. San José: Ministerio de Salud.

Mitchell, Kirstin, Kaye Wellings y Maria Zuurmond. 2012. "Young People". En Sexual Health. A Public Health Perspective, editado por Kaye Wellings, Kirstin Mitchell, Martine Collumbien, 73-84. New York: Open University Press.

OMS. 2006. Defining sexual health. Report of a technical consultation on sexual health 28-31- January 2002. Ginebra: Organización Mundial de la Salud.

PEN-INEC. 2013. Indicadores Cantonales. Censos Poblacionales 2010-2011. San José: Proyecto Estado de la Nación, Instituto Nacional de Estadística y Censos.

Rodríguez, Julieta. 2014. "El talón de Aquiles de las políticas públicas." IV Simposio Internacional y VIII Nacional sobre Derechos de la Niñez y Adolescencia: ¿Cómo garantizar la protección integral ante las transformaciones de la política pública en América Latina y el Caribe? San José: UCR.

UNFPA. 2011. Específicamente, se hizo referencia a materiales producidos por el Programa Conjunto Giro 180, tales como el Modelo de Promoción de Estilos de Vida Saludables y Prevención del VIH y SIDA entre Adolescentes. San José: Fondo de Población de las Naciones Unidas.

Vasilachis, Irene. 2014. Discurso científico, político, jurídico y de resistencia. Análisis lingüístico e investigación cualitativa. Barcelona: Gedisa. 